\title{
Le Burkina Faso et la Fondation Nature et Vie ont su brillamment relever le défi du forum Planèt'ERE
} 3

Forum du 18 au 26 juillet 2005 - Ouagadougou, Burkina Faso

Robert Litzler

\section{(2) OpenEdition}

\section{Journals}

Édition électronique

URL : http://journals.openedition.org/ere/4322

DOI : 10.4000 /ere.4322

ISSN : 2561-2271

Éditeur

Centr'ERE

Référence électronique

Robert Litzler, «Le Burkina Faso et la Fondation Nature et Vie ont su brillamment relever le défı du forum Planèt'ERE 3 », Éducation relative à l'environnement [En ligne], Volume 5 | 2005, mis en ligne le 20 novembre 2005, consulté le 24 septembre 2020. URL : http://journals.openedition.org/ere/4322 ; DOI : https://doi.org/10.4000/ere.4322 


\section{Le Burkina Faso et la Fondation Nature et Vie ont su brillamment relever le défi du forum Planèt'ERE}

3

Forum du 18 au 26 juillet 2005 - Ouagadougou, Burkina Faso

Robert Litzler

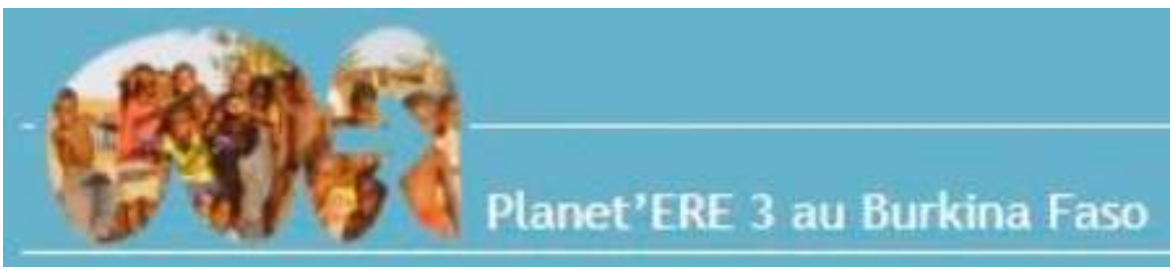

1 Le 23 novembre 2001, dans la majestueuse salle de l'UNESCO, un moment magique suivi d'une salve d'applaudissements : devant 1500 acteurs de l'éducation relative à l'environnement (ERE), l'Afrique francophone annonce qu'elle sera l'hôte du forum Planèt'ERE 3. Ainsi, après Montréal (novembre 1997) et Paris (novembre 2001), une ville d'Afrique accueillera ceux et celles qui croient qu'une éducation préoccupée de la relation avec l'environnement est le plus sûr chemin pour espérer un cadre de vie meilleur pour chaque citoyen de la planète.

2 Nul ne savait alors quel serait le pays qui serait en mesure de relever ce défi, mais lorsqu'en novembre 2003, la Fondation Nature et Vie, épaulée puissamment par le ministère de l'Environnement du Burkina Faso, fit parvenir sa candidature au comité de sélection, nous n'avions pas de doute que ce partenariat entre une ONG de la société civile et l'appareil gouvernemental du Burkina Faso serait une combinaison gagnante. Dès lors, Ouagadougou est apparu comme la ville africaine qui aurait l'honneur de recevoir le forum Planèt'ERE 3. Autre facteur rassurant : ce gouvernement et cette ville n'avaient-ils pas donné au monde entier la preuve de leur capacité d'organiser des 
événements d'envergure mondiale ? Le Burkina Faso n'était-ce pas le pays choisi pour organiser le Xe sommet des chefs d'État de la Francophonie?

3 Si sur le plan administratif et logistique, la collectivité internationale des éducateurs à l'environnement n'avait aucune raison de douter de la capacité de la Fondation Nature et Vie d'organiser le forum Planèt'ERE 3. Elle avait cependant la responsabilité d'épauler de façon significative la commission chargée de construire le contenu et le calendrier des activités de l'événement. Les acteurs québécois et français étaient évidemment interpellés en première ligne, mais tous ceux qui avaient vécu les deux premiers événements étaient aussi ciblés comme d'éventuelles personnes ressources. Il était aussi évident qu'une présence nombreuse au forum Planèt'ERE 3 des acteurs du Nord serait souhaitée, le savoir-faire et les expertises des acteurs en ERE de ces pays constituant un apport incontournable au succès du forum.

4 Une autre question demeurait préoccupante. L'organisation d'un forum Planèt'ERE dans un pays d'Afrique posait par ailleurs clairement la question de la capacité financière de ce pays de faire face aux dépenses. Cette question s'était posée dès le lendemain de la proclamation de l'Afrique de vouloir organiser le forum Planèt'ERE 3. Aussi, la solution envisagée pour soulager financièrement le pays hôte passait par la création d'une organisation internationale Planèt'ERE, qui, une fois qu'elle aurait une existence légale, pourrait avoir accès aux programmes financés par les grandes institutions financières internationales. Ce n'était évidemment pas la seule motivation, la principale étant d'offrir à tout acteur en ERE un lieu d'appartenance à la grande famille francophone de l'ERE.

5 Les membres du Collectif International Planèt'ERE (CIP) créé à l'occasion du Forum Planèt'ERE 1 et qui ont poursuivi leurs travaux depuis lors peuvent éprouver une légitime fierté d'avoir réussi cette opération le 16 avril 2004, au Muséum National d'Histoire Naturelle de Paris, au lendemain du Colloque international sur « l'Éducation à l'Environnement pour un Développement Durable ». Ce jour-là, 80 membres fondateurs de 16 pays ayant le français en partage créaient l'organisation internationale non gouvernementale (OING) Planèt'ERE et faisaient du Collège de Rosemont à Montréal son siège social provisoire jusqu'à l'assemblée générale statutaire qui aurait lieu durant le forum Planèt'ERE 3. On peut comprendre que c'est la prudence qui a commandé aux membres fondateurs de vouloir éprouver la capacité du

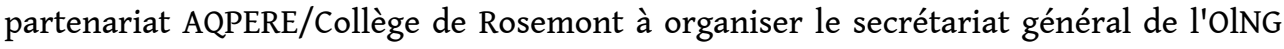
Planèt'ERE. Ce caractère provisoire fut toutefois une erreur stratégique, car l'obtention d'une incorporation légale ne s'accommode pas d'un siège social provisoire. Cela avait comme conséquence de rendre impossible la recherche de financement au bénéfice de la Fondation Nature et Vie pour le Forum Planèt'ERE 3.

6 Nous voilà donc le 18 juillet 2005, à la cérémonie d'ouverture du troisième Forum Planèt'ERE en présence du Président du Burkina Faso, du président de la République du Mali, du Premier Ministre du Niger, du Représentant du Président de l'Union Africaine, $\mathrm{du}$ Représentant du Secrétaire général de l'Organisation Internationale de la Francophonie, du Maire de la ville de Ouagadougou, du Président du Comité organisateur du forum Planèt'ERE 3, de la Présidente de la Fondation Nature et Vie, du Président de l'OING Planèt'ERE, auxquels s'étaient joints les membres du gouvernement du Burkina Faso, des présidents des institutions, des chefs de missions diplomatiques, des représentants des organisations internationales, tous visiblement fiers qu'un pays africain ait su relever, et avec quel brio, le défi de rassembler sur le magnifique site du 
Salon International de l'Artisanat de Ouagadougou et sur treize sites régionaux en province, 2421 acteurs de l'éducation relative à l'environnement venus de 43 pays des cinq continents et dont la très grande majorité était issue de la société civile.

7 Fiers et émus nous l'étions également tous pour rappeler avec force le rôle que doit jouer l'ERE dans les politiques et les stratégies de développement des gouvernements pour une nouvelle vision du monde et de sa gestion, intégrant un développement économique soutenable, socialement équitable et écologiquement viable.

8 Si nous étions rassemblés ce 18 juillet 2005 au Salon International de l'Artisanat de Ouagadougou, c'était pour réaffirmer que l'éducation relative à l'environnement a pour mots clés l'éthique, l'équité, la solidarité, le partage, le respect de l'autre dans la diversité, des valeurs essentielles pour faire prendre conscience aux générations présentes et futures, que les ressources de la planète appartiennent à tous ; qu'il revient à chacun de nous de défendre cette vérité, à l'école, dans nos familles, sur notre lieu de travail et dans nos instances de décision.

9 À ceux qui nous questionnent sur le sens de notre action, nous répondons que l'éducation relative à l'environnement doit entraîner chez celui qui la vit un réflexe de responsabilité personnelle et l'adoption de comportements quotidiens plus respectueux des ressources de la planète. Nous leur disons qu'aucune stratégie vers le mieux-être de l'humanité ne peut connaître le succès, sans une participation massive et consciente de tous les humains qui peuplent notre planète, que cela nous conduit à l'obligation de participer à la vie politique de nos territoires, pays et régions ; que cela nous force à adopter des comportements marqués d'une sociabilité respectueuse de tous les droits humains et nécessaires aux combats engagés pour vaincre la pauvreté, les oppressions de toute nature et pour inverser la spirale des déséquilibres qui ruinent l'aspiration des peuples à leur mieux-être ; que c'est une œuvre gigantesque dans laquelle nous devons tous nous engager sans retard.

10 Pour cela, il nous faut être inventifs et solidaires pour résoudre les problèmes auxquels nous sommes confrontés, dans un esprit de concertation et de cohésion, de coopération et de concorde. L'environnement est une problématique préoccupante pour toute l'humanité et la paix, la stabilité durable et l'équité resteront à l'état de mots sans la prise en compte des questions environnementales dans nos politiques de développement et de coopération aux plans national, régional et international. Chacun doit jouer sa partition. Les acteurs engagés sur le chantier éducatif l'ont tous compris et ont en commun de privilégier la mise en œuvre d'actions participatives. L'heure est maintenant venue de nous mettre à l'œuvre. Alors, bâtissons ensemble un avenir meilleur, rempli de chaleur humaine où il fera bon vivre pour tous. Il nous appartient maintenant et tout de suite de traduire en actes concrets, les résolutions, les recommandations et les décisions réaffirmées à Ouagadougou. Elles sont basées sur la place privilégiée que doit occuper l'être humain pour qu'il y ait un véritable avenir viable pour tous.

11 Le 26 juillet 2005, dans le Salon International de l'Artisanat de Ouagadougou, l'OlNG Planèt'ERE s'est donnée comme présidente pour les quatre prochaines années Araba Kadiatou Yonli, présidente de la Fondation Nature et Vie, à qui la grande famille des acteurs de l'ERE doit le succès du forum Planèt'ERE 3. Le Collège de Rosemont de Montréal, quant à lui, s'est vu confirmer l'attribution du siège social de l'OING Planèt'ERE par l'Assemblée générale, ce qui permettra maintenant de lui donner une existence légale. C'était un des défis que les 38 membres de la délégation canadienne 
avaient à relever. Il appartient maintenant à tous les acteurs de l'ERE d'en faire un opérateur écouté et apprécié de la décennie 2005-2014 dédiée à l'éducation au développement durable.

\section{AUTEUR}

\section{ROBERT LITZLER}

Président de l'AQPERE (Association québécoise pour la promotion de l'éducation relative à

l'environnement) 